\title{
Conocimiento profesional del docente en educación superior: una confrontación entre lo real y lo deseado
}

\author{
Antonio-Manuel Rodríguez-García y Nazaret Martínez-Heredia
}

\section{RESUMEN}

Este trabajo tiene un doble objetivo: por un lado, analizar la presencia de las diferentes dimensiones del conocimiento profesional docente en el desempeño de la profesión y, por otro, valorar la importancia que futuros profesionales de la educación otorgan a la necesidad de presentar adecuadamente tal conocimiento. Se ha seguido una metodología cuantitativa empleando el cuestionario como instrumento de recogida de información. La muestra ha estado compuesta por 89 alumnos del cuarto curso del grado de Pedagogía de la Universidad de Granada. Los resultados indican un panorama deficitario para el desempeño profesional del docente en el aula como modelo de referente en la formación de los futuros formadores. En este sentido, se observa que los alumnos conceden en todas las dimensiones una mayor importancia al conocimiento en contraposición con el nivel de presencia que han experimentado a lo largo del transcurso de su carrera universitaria.

Palabras clave: formación del profesorado, educación superior, conocimiento profesional docente, práctica educativa, España.

\section{Antonio-Manuel Rodríguez-García}

arodrigu@ugr.es

Español. Licenciado en Pedagogía por la Universidad de Granada, España. Profesor Sustituto Interino en el Departamento de Didáctica y Organización Escolar de la Facultad de Educación, Economía y Tecnología (Campus de Ceuta) de la Universidad de Granada. Principales temas de investigación: desarrollo profesional docente, tecnología educativa, competencia digital, educación, aprendizaje a lo largo de la vida.

\section{Nazaret Martínez-Heredia}

nazareth@ugr.es

Española. Licenciada en Pedagogía por la Universidad de Granada, España. Contratada FPU (Formación de Profesorado Universitario) en el Departamento de Pedagogía de la Universidad de Granada, España. Temas de investigación: desarrollo profesional del docente, pedagogía/educación social, educación para la muerte, educación en personas adultas y mayores, educación intergeneracional. 


\section{Conhecimento profissional do docente em educação superior: uma confrontação entre o real e o desejado}

\section{RESUMO}

Este trabalho tem um duplo objetivo: por um lado, analisar a presença das diferentes dimensões do conhecimento profissional docente no desempenho da profissão e, por outro, observar a importância que futuros profissionais da educação dão à necessidade de apresentar adequadamente tal conhecimento. Se seguiu uma metodologia quantitativa empregando o questionário como instrumento de coleta de informação. A amostra ficou composta por 89 alunos do quarto curso de Pedagogia da Universidad de Granada. Os resultados indicam um panorama deficitário para o desempenho profissional do docente na sala de aula como modelo de referência na formação dos futuros formadores. Neste sentido, se observa que os alunos concedem em todas as dimensões uma maior importância ao conhecimento em contraposição com o nível de presença que têm experimentado ao longo do transcurso de sua carreira universitária.

Palavras chave: formação do professorado, educação superior, conhecimento profissional docente, prática educativa, Espanha.

\section{Professional Knowledge of Teachers in Higher education: A Confrontation Between Reality and Desire}

\section{ABSTRACT}

This work has a double objective: on one hand, to analyze the presence of the different dimensions of professional teaching knowledge in the performance of the profession and, on the other hand, to value the importance that future education professionals attach to the need to adequately present such knowledge. A quantitative methodology has been followed, using the questionnaire as an instrument for collecting information. The sample consisted of 89 fourth-year students of a Pedagogy degree at the University of Granada. The results indicate a deficit panorama for the professional performance of the teacher in the classroom as a model of reference in the training of future trainers. In this sense, the author observed that students in all dimensions grant greater importance to knowledge in an inverse proportion to the level of presence they have experienced throughout the course of their university career.

Key words: teacher training, higher education, teaching professional knowledge, educational practice, Spain. 


\section{Introducción}

La educación precisa de profesores altamente capacitados para fomentar en el alumnado una actitud crítica y compleja y formar a las futuras generaciones y profesionales de diferentes campos. En este sentido, numerosos autores han señalado la existencia de diferentes categorías de conocimiento y han ofrecido múltiples clasificaciones destinadas a perfilar los distintos componentes que conforman el conocimiento base para la enseñanza. Por ello, es necesario entender el desarrollo profesional del docente como un proceso flexible, personal y evolutivo (Marcelo, 2014). A pesar de haber diferentes clasificaciones, Vera, Torres y Martínez (2014) exponen tres dimensiones dentro del conocimiento profesional docente: dimensión personal, contextual y de conocimiento y destrezas. Esta última parte aborda tres elementos relacionados con los aspectos didácticos del trabajo en el profesorado: conocimiento didáctico del contenido, destrezas en la gestión dentro del aula y destrezas docentes.

Por otro lado, López-López (2009) afirma que los profesores deben contar con una diversidad de conocimientos que les sirven para orientar su práctica, tales como: el conocimiento de uno mismo, el ambiente para la enseñanza, la materia, así como el desarrollo del currículo y de la instrucción. Otros autores señalan, por su parte, siete categorías diferentes: conocimiento del contenido, pedagógico, del currículo, del aprendiz y del aprendizaje, del contexto, pedagógico del contenido y de la filosofía, metas y objetivos educativos. Históricamente, Grossman (1990) limitó este intento de categorización a cuatro componentes fundamentales: conocimiento de la materia, pedagógico general, didáctico del contenido y del contexto. Solís et al. (2012), por su parte, distinguen entre el conocimiento inicial y el deseable que debe utilizarse como meta o referencia y que se va construyendo a través de la formación continua, reflexión y experiencia.

En el ámbito iberoamericano, la aportación de Marcelo (2014) nos acerca a cuatro tipos de conocimiento del profesor necesarios para la enseñanza y que coinciden en gran medida con los ya reflejados anteriormente por Grossman (1990) y Shulman (1999): conocimiento del contenido, pedagógico general, didáctico del contenido y del contexto. Por este motivo, para la realización de la presente investigación hemos centrado nuestra atención en esta última clasificación, la cual procedemos a delimitar a continuación.

\section{Conocimiento profesional docente: una breve introspección}

Atendiendo al conocimiento de la materia, Shulman (2005) afirmaba que para su dominio se exige ir más allá de los conceptos básicos de una materia hacia una comprensión de las estructuras que la conforman. Guerra y Montanegro (2017) consideran que este conocimiento, además de incluir los hechos y conceptos de una disciplina, también incorpora la comprensión de los métodos y reglas que guían el estudio de la misma y, en este caso, incluyen las siguientes categorías:

- Conocimiento del contenido para la enseñanza, que se refiere al conocimiento de hechos, conceptos y principios de la materia.

- Conocimiento sustantivo para la enseñanza, que es aquel que guía la investigación en el campo y permite interpretar los datos.

- Conocimiento sintáctico para la enseñanza, que nos aproxima a las vías que permiten la integración del nuevo conocimiento en el ya existente.

- Creencias acerca de la materia, que engloba a creencias y orientaciones sobre la materia.

Depaepe, Verschaffel y Kelchtermans (2013) asumen que se trata de un intento por superar la distinción existente entre pedagogía y contenido, enfatizando en el rol que posee dicha asignatura entendida como contenido en la enseñanza y en la formación del profesorado. 
También es importante destacar a Schwab (1978), citado por Fernández Cruz (2006), el cual describe el conocimiento de la materia como una estructura de la disciplina situada en una doble dimensión: conocimiento sustantivo y conocimiento sintáctico. El primer conocimiento se refiere al cuerpo interrelacionado de conceptos de una materia, mientras que el segundo hace referencia a los métodos que se usan en la disciplina para construir conocimiento. Borrero y Losada (2012), por su parte, exponen que el contenido debe reorganizarse y transformarse teniendo en cuenta las características de los alumnos, del contexto y del curriculum. Así pues, para enseñar un concepto no solamente es importante dominar el contenido y poseer conocimientos generales de pedagogía, sino también conocimientos específicos respecto a la enseñanza de dicho contenido (Vergara y Cofré, 2014).

En definitiva, este tipo de conocimiento tiene que ver con el dominio, por parte del profesor, de los paradigmas de investigación en cada disciplina, el conocimiento en relación con cuestiones como la validez, tendencias y perspectivas en el campo de la especialidad.

De otro lado, al hablar de conocimiento pedagógico general hacemos referencia no sólo al mero conocimiento de los hechos o conceptos, sino más bien a la interrelación que se establece entre la pedagogía y el contenido de la materia, de forma que se genere una mayor comprensión por parte de los alumnos (Vicedo, 2015). Según Köing et al. (2014) este conocimiento está integrado por varias teorías que permiten aproximarnos a la enseñanza, estudiar su evolución, técnicas instructivas y su uso en clase, habilidades de ejecución: voz, movimientos, estructura de la acción y cómo hacer un plan para la misma, estrategias interactivas y su uso; técnicas de evaluación y su uso, y técnicas de dirección en clase.

En otras palabras, este tipo de conocimiento respondería al cómo enseñar. Por ello, incluye el conocimiento de los principios y estrategias de la gestión y la organización de la clase que facilitan la impartición de la materia significativamente. Debe incluir una comprensión general de las teorías de la educación y un conocimiento general sobre el alumnado, además de los principios de gestión y organización de la clase. Por ello, este tipo de conocimiento se circunscribe al conocimiento de los alumnos, del $\mathrm{cu}^{-}$ rriculum, de la instrucción y de la evaluación.

El conocimiento del curriculum incluye el saber de los materiales curriculares y programas que sirven de herramientas al profesor durante el desarrollo del curriculum (Shulman y Shulman, 2004). Stenhouse (2010) considera que el curriculum sirve para comunicar los rasgos y principios esenciales de un propósito educativo para que pueda ser trasladado efectivamente a la práctica y a la reflexión. Como bien nos explican Latorre Medina, López López y Rodríguez Entrena (2009), se trata de la intersección entre contenido de la materia per se, y los principios didácticos generales mostrados como válidos para una enseñanza efectiva. Por ello, podría construirse con y sobre el conocimiento del contenido, pedagógico y de los alumnos.

Stabback (2016) explica que moverse en este espacio común, exige una comprensión profunda, flexible y abierta del contenido, comprendiendo las dificultades más probables que tendrán los alumnos con estas ideas, comprendiendo las variaciones de los métodos y modelos de enseñanza para ayudar a los alumnos en su construcción del conocimiento.

Respecto al conocimiento de los contextos educativos, éste incluye el conocimiento amplio de la sociología de la clase, el funcionamiento de la administración escolar y la configuración social y cultural del entorno del centro y se encuentra relacionado con las orientaciones, las estrategias, y los estudiantes (Osorio, Hernández, Soto y Orozco, 2014). Bermúdez y Longui (2012), además, afirman que el conocimiento del contexto lleva consigo un conocimiento situacional referente al medio sociocultural, histórico, ambiental e institucional, un conocimiento de la lengua y 
un conocimiento mental de alumnos y docentes. Por otro lado, Marcelo y Vaillant (2015) señalan que este tipo de conocimiento incluye el conocimiento de la escuela, de su cultura, del profesorado y de las normas de funcionamiento. La importancia de este conocimiento queda avalada por el hecho de que el docente debe reflexionar sobre las peculiaridades de su contexto y desarrollar acciones de transformación (Fandiño-Parra, 2017).

Atendiendo al conocimiento de los alumnos, según Fernández Cruz (2006), está compuesto por conocimiento general sobre alumnos y sobre determinados aspectos de la interacción con ellos. Barrón (2015) señala la importancia de conocer las habilidades del alumnado, así como sus elaboraciones y significados en torno a los contenidos propuestos. Por ello, es sumamente importante usar un método pedagógico distinguido por la variedad de técnicas y recursos para establecer una comunicación que favorezca la adaptación al entorno social y cultural en el grupo de estudiantes (García-Cano et al., 2017). Los profesores deben ser capaces de identificar las fortalezas y debilidades, así como trabajar con los alumnos que tengan necesidades específicas de apoyo educativo.

Por último, el conocimiento didáctico del contenido integra en un solo ámbito aspectos propios de la disciplina académica y de la pedagogía o la didáctica general. Dicho contenido incluye conexiones entre el conocimiento didáctico de la materia y del profesor, para poder permitir una transformación del contenido en su enseñanza (Díaz et al., 2016). En esta línea, Bazán y Zupa (2016) concretan una serie de pasos para la transformación del contenido en entendible, éstos son: conocimiento comprensivo de la materia, transformación del conocimiento científico enseñable, fase interactiva de enseñanza, estructuración del proceso, reflexión sobre el proceso, la aproximación (auto)biográfico-narrativa como marco conceptual de análisis del conocimiento docente. En definitiva, se trata de un conocimiento que capacita al docente para organizar el contenido académico en un discurso narrativo de enseñanza comprensivo para los alumnos.

Llegados a este punto, debemos mencionar que una de las mayores problemáticas dentro del ámbito universitario es la incongruencia existente entre la teoría y la práctica del profesionalismo docente, es decir, entre lo que se dice que es lo correcto y lo que realmente se hace. En este sentido, cuando los profesores de la Facultad de Ciencias de la Educación de la Universidad de Granada (UG) pretenden enseñar a sus alumnos a ser buenos profesionales de la educación, ha de haber una correcta relación entre el conocimiento profesional mostrado por el docente y el que pretenden enseñar a los futuros profesionales de la educación, es decir, una congruencia entre la "teoría" y la "puesta en práctica", como modelos de referencia para los futuros profesionales de la educación. Por tanto, creemos necesario analizar los conocimientos propios de la profesionalidad docente desde el punto de vista de los estudiantes ya que, al fin y al cabo, son quiénes mejor pueden determinar su presencia o ausencia, puesto que han estado trabajando con ellos durante, al menos, cuatro años consecutivos.

\section{Metodología}

El objetivo general de nuestra investigación es doble. Por un lado, nos centramos en analizar el grado de presencia o ausencia de los conocimientos que definen la profesionalidad docente de los profesores de la Facultad de Ciencias de la Educación (FCE) de la UG (España) según la opinión de sus propios educandos. Por otro lado, este trabajo también tiene por objetivo analizar la importancia que los futuros docentes le conceden a presentar o no dicho conocimiento en el ejercicio de la docencia.

Para la consecución de las metas propuestas, partiremos de una metodología cuantitativa, la cual nos ayudará a cuantificar y analizar la información para así establecer posteriores comparaciones entre lo que los alumnos han visto como referencia en sus 
profesores y el grado de importancia que ellos le conceden a la presencia de dicha dimensión.

La investigación fue desarrollada en el curso 2017-2018. La muestra participante de nuestro estudio ha estado formada por los alumnos del cuarto curso del grado en Pedagogía de la FCE de la UG. Se ha seguido un muestreo aleatorio y estratificado. El total de la población estaba compuesta por 102 estudiantes matriculados en este curso de la titulación. Dado el número reducido de alumnos, se optó por invitar a toda la población de forma que todos tuviesen la oportunidad de ser sujetos participantes. De este modo, se obtuvo una muestra final de $n=89$ sujetos (91\% mujeres y $9 \%$ hombres), por lo que tratamos con una muestra significativa superando los sujetos necesarios de la muestra calculados a través de un intervalo de confianza del 95\% y cuyos resultados precisaban la participación de, al menos, 81 estudiantes. La elección de esta población se debe, principalmente, a la experiencia de los alumnos tras haber cursado tres años en la FGE y, por tanto, poseer una madurez terminológica y una actitud crítica para ayudarnos a solventar nuestro problema de investigación. En este sentido, como futuros profesionales del sector, han de conocer y haber visto la evolución de los conocimientos que pretendemos medir en los docentes que han tenido a lo largo de estos tres años.

Para la recogida de información hemos utilizado un cuestionario construido ad hoc, de escala Likert ( 1 completamente en desacuerdo/nunca; 2 en desacuerdo/pocas veces; 3 de acuerdo/la mayoría de las veces; y 4 completamente de acuerdo/siempre, o casi siempre) validado por medio de un juicio de expertos, donde los alumnos valorarán, por un lado, la presencia o ausencia de una serie de afirmaciones correspondientes al conocimiento profesional docente, es decir, conocimiento pedagógico, didáctico, de la materia, de los alumnos, del contexto y del curriculum, que ellos han observado a lo largo del transcurso de su titulación y, por otro lado, el grado de importancia que ellos conceden a la puesta en práctica de dicho conocimiento en el desempeño de la actividad profesional docente. La validación del cuestionario se realizó siguiendo las siguientes pautas:

- Definición del objetivo del juicio de expertos para validar el cuestionario "Conocimiento Profesional Docente".

- Selección de cinco expertos pertinentes teniendo en cuenta los criterios definidos anteriormente, considerando su formación académica y experiencia profesional.

- Evaluación por medio de los expertos atendiendo a la pertinencia, claridad y adecuación de los indicadores del cuestionario utilizando una planilla.

- Una vez obtenidos los resultados se calculó la concordancia entre jueces y finalmente se elaboraron unas conclusiones atendiendo a la descripción psicométrica de la prueba.

Una vez realizado el juicio de expertos, el cuestionario recoge aspectos correspondientes a los diferentes conocimientos propios del conocimiento profesional docente. De una manera más detallada, recogemos dicha información en la tabla 1 , donde podemos observar los ítems que corresponden a cada conocimiento.

Posteriormente se realizó una prueba de fiabilidad mediante el alfa de Cronbach, obteniendo un índice de $a=0.896$, dato que le confiere un elevado grado de coherencia $(89.6 \%)$ debido su proximidad a la unidad (Rodríguez y Valldeoriola, 2009). 
Tabla 1: Cuestionario de evaluación del profesionalismo docente de la Facultad de Ciencias de la Educación

\begin{tabular}{|c|c|}
\hline Conocimientos & ítems \\
\hline Conocimiento pedagógico general & $1-8$ \\
\hline Conocimiento del curriculum & $9-16$ \\
\hline Conocimiento de la materia & $17-22$ \\
\hline Conocimiento del contexto & $23-26$ \\
\hline Conocimiento de los alumnos & $27-31$ \\
\hline Conocimiento didáctico del contenido & $32-36$ \\
\hline
\end{tabular}

\section{Resultados}

A continuación, presentamos los resultados obtenidos atendiendo a cada una de las dimensiones del cuestionario. En este sentido, se muestran una serie de gráficas comparativas que analizan cada atributo propio del conocimiento profesional docente en función de la presencia mostrada (primera barra) y la importancia recibida por parte de los estudiantes (segunda barra).

En primer lugar, haciendo alusión al conocimiento pedagógico general, se ha obtenido una media de presencia total de 2.66 sobre 4, por lo que el alumno considera que sus docentes suelen presentar este tipo de conocimiento algunas veces en sus clases. Sin embargo, señalan la necesidad de demostrar mayormente este contenido reconociéndole una importancia total de 3.66 sobre 4 , por lo que consideran que los docentes deberían hacer mayor hincapié en mostrar y enseñar principios y estrategias relacionadas con el empleo de diferentes teorías de aprendizaje para mejorar el proceso de enseñanza y hacer más interesante la asignatura (I1); usar técnicas pedagógicas que mejoren el ambiente, el clima y la relación entre profesor-alumno (I2); optimar la organización y planificación de las clases (I3); marcar un ritmo de clase que permita seguir sus explicaciones (I4); mostrar interés por dar explicaciones claras empleando diferentes tonos de voz y movimientos corporales (I5); motivar a los estudiantes para participar crítica y activamente en el transcurso de la materia (I6); así como posibilitar hablar con ellos acerca de la materia fuera de clase (I8). El ítem 7, por su parte, es aquel que presenta una media mayor en la importancia concedida, ya que los estudiantes consideran trascendental que el profesorado proporcione ayuda y asesoramiento acerca de la forma más eficaz de estudiar la asignatura. Finalmente, señalar que, pese a que los alumnos del $4^{\circ}$ grado de Pedagogía conceden una importancia fundamental al empleo por parte del docente de diferentes técnicas pedagógicas que mejoren el clima general del aula, las relaciones entre los alumnos y con el profesor y que terminen por proporcionar un clima eficaz y acogedor, señalan que pocas veces han observado esta situación en los docentes que han tenido a lo largo del transcurso de su carrera universitaria (figura 1). 


\section{Figura 1. Conocimiento pedagógico:} presencia/ausencia vs importancia

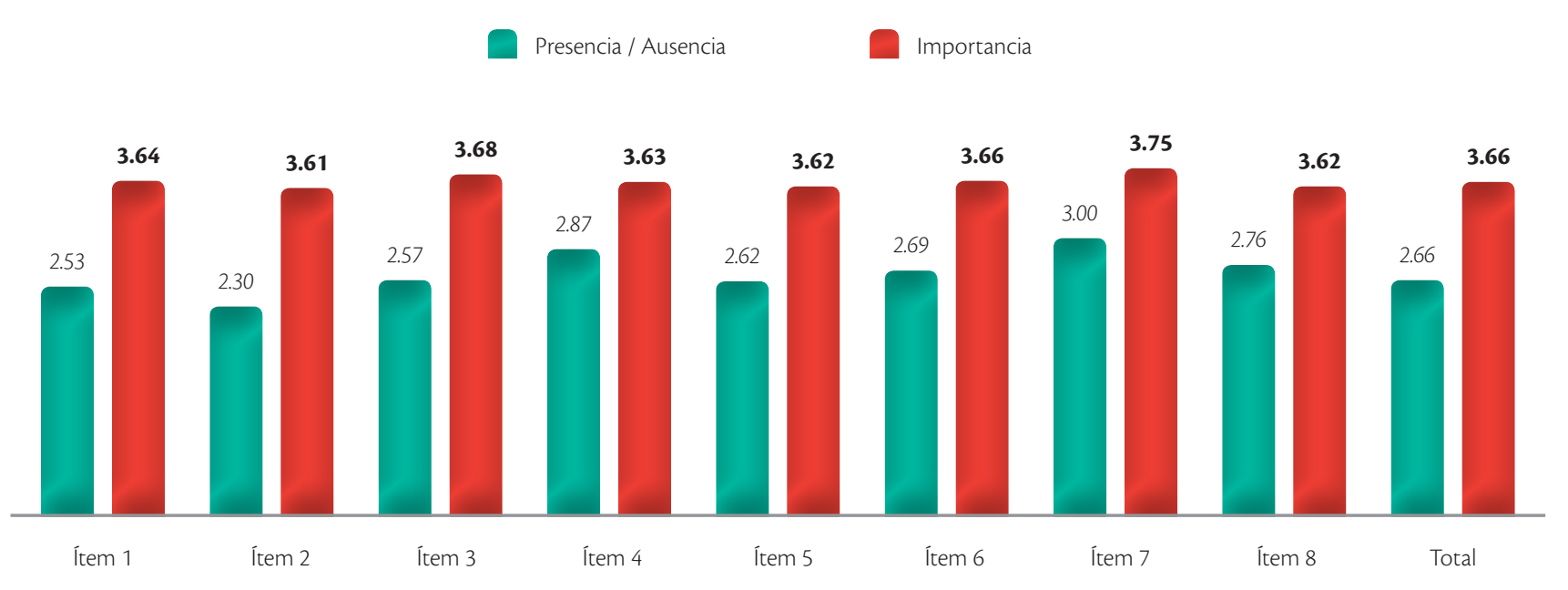

En segundo lugar, atendiendo al conocimiento del curriculum, con relación a la presencia o ausencia de esta dimensión, hemos hallado una media total de 2.71 sobre 4, siendo el ítem 11 (figura 2) al que más presencia se le otorga y el ítem 15 al que menos. El ítem 11 hace referencia a que los profesores dan a conocer el programa de su asignatura explicando en todo momento los objetivos, las competencias a conseguir, los contenidos que se van a desarrollar, los métodos de evaluación... En cambio, el alumnado considera que el ítem 15, que se refiere a si el docente renueva los métodos y contenidos de enseñanza y los adapta a las necesidades de sus alumnos, se da muy pocas veces.

Sin embargo, la importancia que se le concede al conocimiento del curriculum presenta una media total de 3.65, por lo que se puede considerar muy importante, a diferencia de la presencia que los estudiantes dicen haber observado en sus docentes de referencia. Se puede deducir que los ítems 11, 12 y 13 (atendiendo a los objetivos, metodología y contenidos de la materia) son los que menos importancia han recibido por parte del alumnado, y ha sido el ítem 13 (exposición clara en clase) el que menor importancia se le ha otorgado por parte de éstos, ya que no comparten de forma unánime el hecho de que en el transcurso de la asignatura se cumpla con lo establecido al principio del curso. Por otro lado, los ítems que han recibido una importancia mayor de este conocimiento han sido el 9 y 10; los cuales se refieren a la preocupación, por parte de los docentes, del aprendizaje de sus estudiantes de manera que proporcionen distintas explicaciones hasta que el contenido se ha entendido por completo (figura 2).

Como se puede observar en la figura 2, existe una diferencia significativa de casi un punto entre la media total de la presencia y la importancia que los alumnos conceden a esta dimensión del conocimiento profesional docente. Esta dimensión, pues, es valorada como muy importante, si bien la presencia con la que ocurre se suele dar en la mayoría de las veces, pero no siempre. El ítem 15 (renovación de metodologías pedagógicas) es aquel en el que la diferencia es muy significativa, ya que se le da una importancia de 3.72 sobre 4, pero la realidad se topa con el hecho de que su presencia es de un 2.29 sobre 4, considerándose que se da pocas veces a lo largo de la carrera. En este sentido, los alumnos afirman que los docentes no se adaptan completamente a las necesidades de sus estudiantes. 


\section{Figura 2. Conocimiento del curriculum:} presencia/ausencia vs importancia

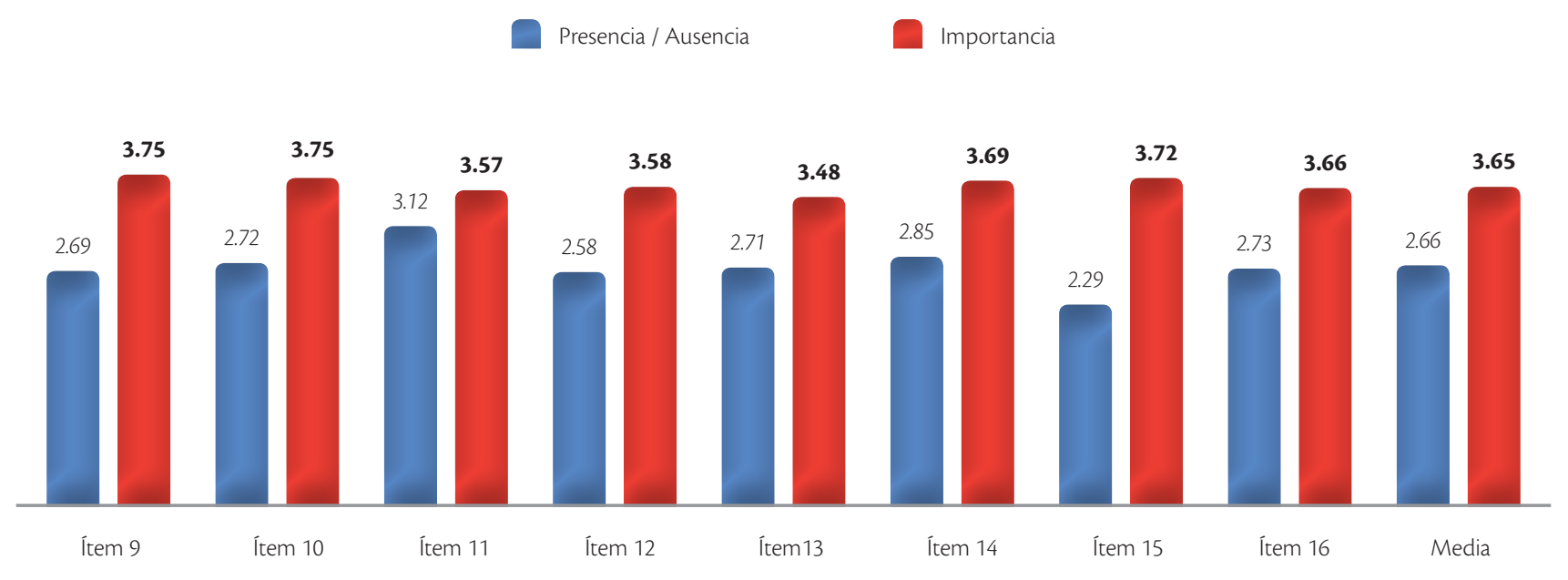

En tercer lugar, respecto al conocimiento de la materia obtenemos como media un valor de 2.67, lo que determina que el alumnado considera que este tipo de atributo se encuentra presente en el profesorado en la mayoría de las ocasiones, a través de diversas acciones docentes: organización de objetivos y contenidos (I17); presentación de un dominio de los fundamentos epistemológicos y paradigmas de investigación de la disciplina que imparte (I18); manifestación de riqueza en los contenidos (I19); establecimiento de interrelaciones con contenidos de otras materias curriculares (I20); exposición de contenidos con ejemplos de investigaciones recientes (I21): así como la consulta y utilización de distintos documentos y propuestas curriculares (I22). Destacamos el ítem 19 con una puntuación muy alta, haciendo referencia a la riqueza de contenido en sus comentarios y juicios.

Por otro lado, en lo que respecta al grado de importancia concedido, en esta dimensión se obtiene una media de 3.52, lo que determina que el alumnado considera que la presencia en el desempeño de la actividad profesional docente del conocimiento de la materia es muy importante. Destaca la relevancia atribuida a la riqueza de contenido en los comentarios y juicios del profesor, considerándolos como algo primordial (figura 3).

Tras la comparación presentada en la figura 3, observamos una diferencia de la media de aproximadamente un punto. El alumnado, pues, piensa que la presencia del conocimiento de los contextos educativos en el desempeño de la actividad profesional docente es importante, e incluso muy importante (con un valor atribuido de 3.52 sobre 4). A su vez, afirman que este tipo de conocimiento se encuentra presente en el profesorado en la mayoría de las veces (con un valor atribuido de 2.67 sobre 4). Estos datos indican que la muestra considera que el conocimiento de la materia por parte del docente es muy importante, pero realmente no se encuentra presente en los docentes tanto como se desearía. 
Figura 3. Conocimiento de la materia: presencia/ausencia vs importancia

Presencia / Ausencia Importancia

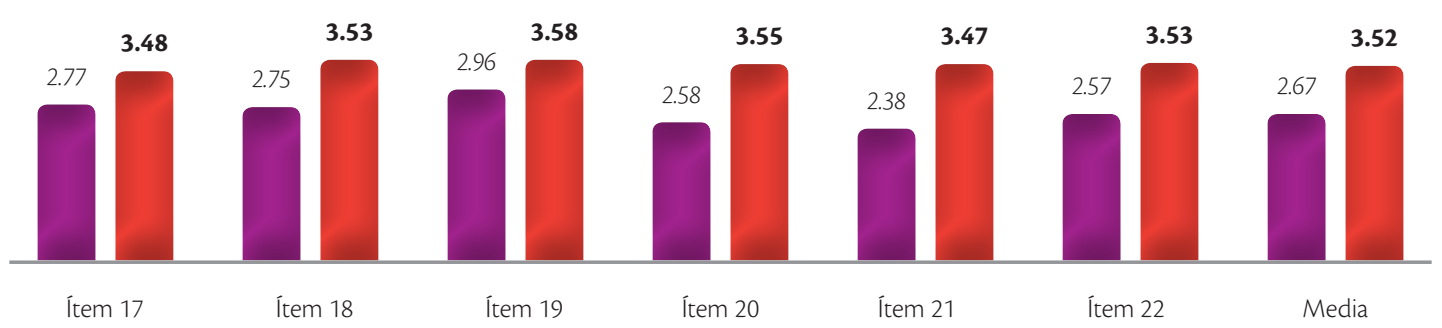

En cuarto lugar, la media obtenida referente al $c^{-}$ nocimiento del contexto es de 2.62, lo que determina que este tipo de conocimiento se encuentra presente en el profesorado en la mayoría de las ocasiones mediante diferentes expresiones: establecimiento de propuestas individualizadas y adecuadas al contexto donde se desarrolla la acción educativa (I23); existencia de una estrecha relación del docente con el entorno educativo y social en el que desarrolla su práctica (I24); esfuerzo en crear un ambiente profesional pero relajado para reforzar el aprendizaje del alumnado
(I25); y constitución de una atmósfera agradable y relajada de trabajo en el aula (I26).

En lo que se refiere a la importancia, y como se viene demostrando a lo largo del presente trabajo, todos los ítems han obtenido una media superior respecto a la relevancia de conocer el contexto donde ejerce su docencia (figura 4) y en los que se valora el establecimiento de propuestas individualizadas adecuadas al contexto donde se desarrolla la acción educativa, la creación de una atmósfera agradable y relajada de trabajo en el aula, etcétera.

Figura 4. Conocimiento del contexto: presencia/ausencia vs importancia

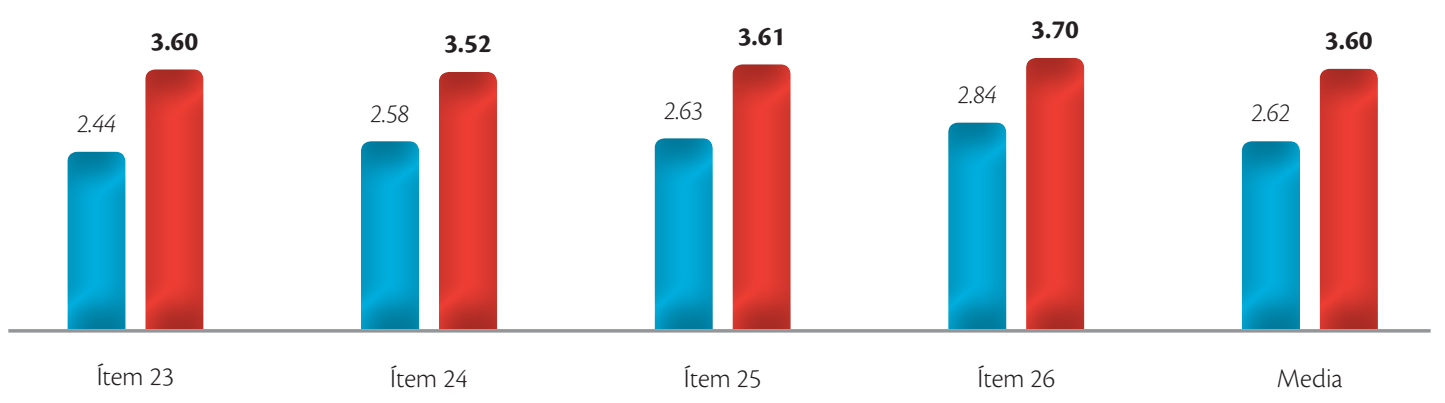


Tras la comparación de los ítems correspondientes a la presencia/ausencia vs importancia del conocimiento del contexto educativo, podemos observar que, como viene mostrándose en esta investigación, la importancia que le conceden los alumnos a los aspectos relacionados con el conocimiento del contexto es mayoritaria frente a la presencia que ellos han observado en sus docentes. Así pues, mientras que el alumnado concede un valor de 3.6 sobre 4 a la importancia de este conocimiento, afirman que han encontrado este conocimiento en sus profesores algunas veces (2.62 sobre 4$)$.

En quinto lugar, haciendo referencia al conocimiento de los alumnos (figura 5) el valor de la media es 2.5, lo que determina que el alumnado considera que este tipo de conocimiento se encuentra presente en algunas o pocas ocasiones, por lo que acciones como la atención a las motivaciones de sus estudiantes (I28); preocupación del profesor por el diálogo y la interacción dentro del aula (I30); o la adaptación a diversas circunstancias de los alumnos (I31) se ven mermados. Destaca, especialmente, la importancia que refiere a que el educador debe adecuarse a aquellos alumnos con necesidades específicas que necesitan una adaptación curricular (I27), así como tener en cuenta motivaciones, puntos de vista, intereses y formas de pensar que posee el alumnado (I29).

\section{Figura 5. Conocimiento de los alumnos: presencia/ausencia vs importancia}

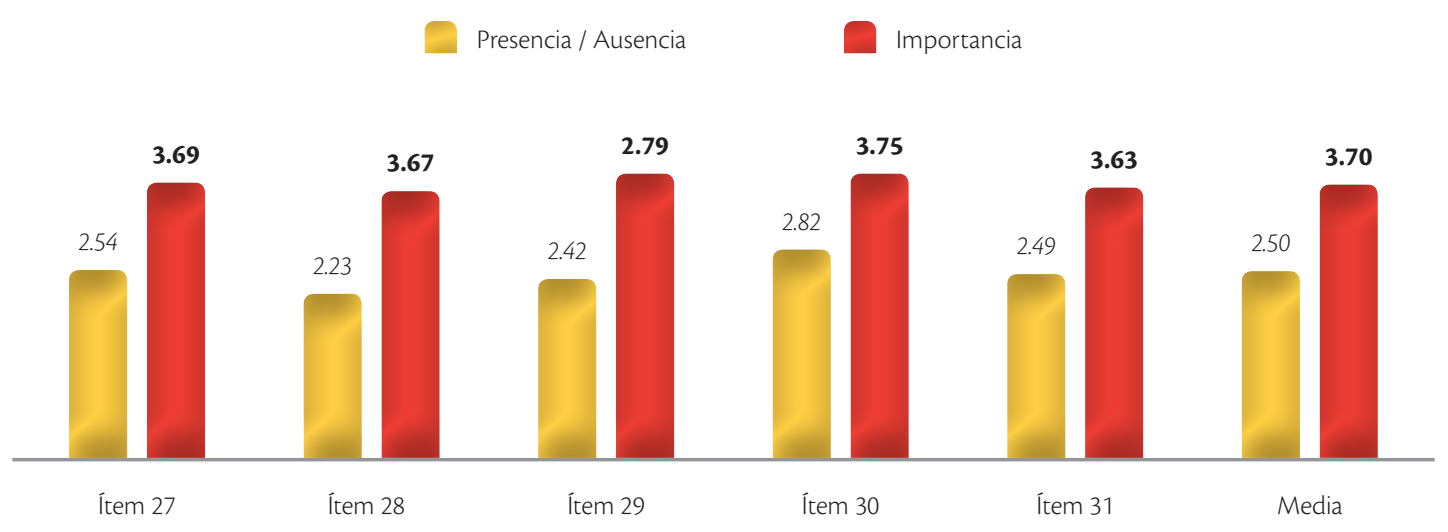

Confrontando los datos de la figura anterior, podemos observar cómo todos los valores referentes a la importancia son superiores a los de presencia en esta dimensión del conocimiento profesional. De este modo, el alumnado considera esencial el hecho de que los profesionales de la docencia se adapten a los estudiantes que presentan necesidades específicas; se adentren, a su vez, en el conocimiento de las motivaciones individuales de los alumnos; tengan en cuenta sus motivaciones; potencien el diálogo y la reflexión entre ellos, etcétera. No obstante, durante el transcurso de su carrera estas acciones no han estado presentes la mayoría de las veces, hecho que se comprueba con la media obtenida referente al total de cada variable estudiada anteriormente.

En último lugar, con relación al conocimiento didáctico del contenido se ha obtenido una media de 2.75 , lo que determina que el alumnado considera que este tipo de conocimiento se encuentra presente en el profesorado en algunas ocasiones a través de diferentes manifestaciones, tales como la proporción de material complementario de la asignatura para mejorar la 
comprensión de los estudiantes (I32); la selección de contenidos útiles para su futuro profesional (I33); la presentación del contenido de una manera asequible (I34); así como el empleo de estrategias para enriquecer el conocimiento del alumnado (I36). Destacamos la puntuación tan alta del ítem 35, el cual intenta conocer la presencia y ausencia ante el fomento del trabajo colaborativo y en equipo con el fin de crear un nuevo conocimiento.
Para finalizar, atendiendo a la figura 6 , se deduce fácilmente la gran importancia que han recibido todos los ítems que tratan de analizar el conocimiento didáctico del contenido dentro del conocimiento profesional docente. En este sentido, los alumnos consideran acciones especialmente relevantes aquellas relacionadas con esta dimensión, tales como: proporción de material complementario; selección de contenidos de utilidad; representación del conocimiento teórico, etcétera.

Figura 6. Conocimiento didáctico del contenido: presencia/ausencia vs importancia

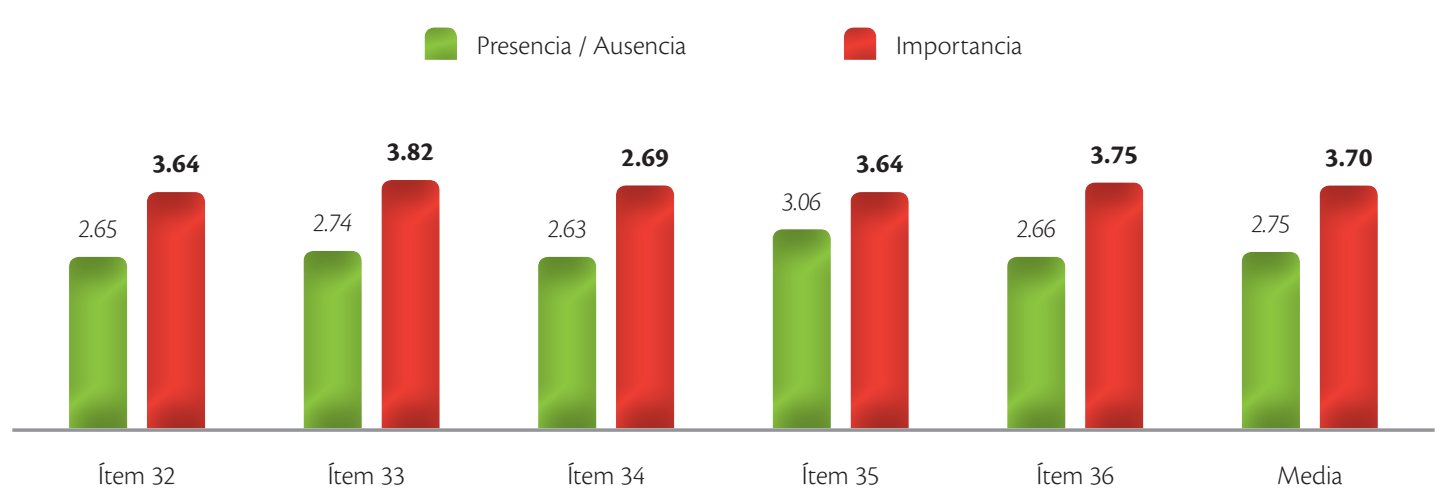

\section{Conclusiones}

Uno de los pilares básicos para el desarrollo profesional docente de aquellas personas que, en un futuro, van a dedicar su labor a la enseñanza, reside en lo que han venido observando en sus clases escolares y universitarias, especialmente a través de sus maestros y profesores como modelos de referencia. El desarrollo de este trabajo pretende incitar a la reflexión acerca de la importancia que tiene el desarrollo de un conocimiento profesional adecuado por parte de los educadores mostrándolo, de esta manera, a las futuras generaciones docentes, pues ello constituye un elemento esencial que este colectivo tomará como referente en la puesta en práctica de su labor (Depaepe et al., 2013; Fandiño-Parra, 2017).
Como hemos podido observar en las páginas que preceden a este apartado, se hace imprescindible, por tanto, actuar de manera lógica y congruente entre lo que los docentes universitarios pretenden transmitir a las futuras generaciones y lo que realmente se hace, es decir, se ha de tratar de mostrar una actuación adecuada entre el profesionalismo docente como teoría y su puesta en práctica en la enseñanza. Esta es la esencia que ha caracterizado nuestra investigación, y en la que nos hemos basado para realizar nuestro análisis. En este sentido, procederemos a realizar una serie de conclusiones, que presentamos en las líneas que prosiguen.

En cuanto al conocimiento pedagógico general, el alumnado que está acabando el último curso de grado, 
considera muy relevante esta dimensión, ya que para ellos es un hecho esencial que los profesores hagan uso de diferentes teorías del aprendizaje para mejorar el proceso de enseñanza, utilicen diferentes técnicas pedagógicas para la mejora del ambiente en clase, el uso de la motivación para que participen de manera crítica y reflexiva, entre otros. No obstante, la presencia y puesta en práctica de este conocimiento en sus docentes, a lo largo del curso de su titulación, no ha sido tan satisfactoria como esperan, en relación con la importancia atribuida a esta dimensión.

En relación con el conocimiento del curriculum, el alumnado perteneciente al cuarto grado universitario considera que se trata de un conocimiento transversal y central para el desarrollo profesional de los docentes, donde se le da una importancia máxima al hecho de que sus alumnos entiendan correctamente los contenidos explicados por el docente. Además, el profesorado ha de tener gran preocupación por el aprendizaje de sus alumnos, así como tener en cuenta las diferentes dificultades de las que parten, entre otros. Sin embargo, al igual que ocurre en la anterior dimensión, la relación presencia/ausencia e importancia no se da de manera proporcional, sino que continúa predominando la importancia concedida por encima del conocimiento mostrado.

Con respecto al conocimiento de la materia, el alumnado considera que no se encuentra presente en el docente tanto como se desearía. Aun así, el profesorado lo suele presentar en los quehaceres del aula, por lo que éste es capaz de organizar objetivos y contenidos, presentar un dominio de los fundamentos epistemológicos y paradigmas de investigación de la disciplina que imparte, manifestar riqueza de contenidos, establecer interrelaciones con contenidos de otras materias curriculares, exponer los contenidos con ejemplos recientes y utilizar distintas propuestas curriculares. Pero, una vez más, la importancia atribuida a este tipo de conocimiento por parte del alumnado es superior, por lo que sería adecuado centrarse en una revisión de la práctica, que atendiese a la voz de los alumnos y la subjetividad de su percepción.

Atendiendo al conocimiento de los contextos educativos observamos que el alumnado considera que la presencia en el desempeño de la actividad profesional docente es muy importante. Asimismo, el alumnado piensa que este tipo de conocimiento se encuentra presente en el profesorado en la mayoría de las veces, por lo que, en cierta medida, éste es capaz de establecer propuestas individualizadas, adecuadas al contexto donde se desarrolla la acción educativa. Por otro lado, existe una estrecha relación del docente con el entorno educativo y social en el que desarrolla su práctica; se esfuerza en crear un ambiente profesional pero relajado para reforzar el aprendizaje del alumnado y posee la capacidad para crear una atmósfera agradable y relajada de trabajo en el aula, destacando este último como la capacidad docente con más presencia dentro del conocimiento de los contextos educativos que presentan los docentes de la Facultad de Ciencias de la Educación. En consecuencia, la importancia atribuida por parte del alumnado a la necesidad de presentar, de una manera adecuada, este tipo de conocimiento es muy alta, pero nuevamente no encontramos una total presencia de éste en la realidad observada por los alumnos de cuarto de grado de la FCE.

Por otro lado, haciendo alusión al conocimiento de los alumnos, como hemos visto anteriormente en nuestro análisis, podemos decir que este tipo de conocimiento se encuentra presente la mayoría de las veces en nuestros profesores, teniendo en cuenta que los alumnos de grado asumen la importancia que tiene la puesta en práctica de éste, como un elemento muy importante en nuestras aulas ya que, como hemos fundamentado en nuestro trabajo, el conocimiento que poseen los profesores sobre sus alumnos y sobre determinados aspectos de la interacción con ellos, incluye numerosos modelos de desarrollo y de aprendizaje que no podemos obviar dentro de nuestras aulas, como pueden ser: la importancia de aspectos 
relevantes de la habilidad del alumnado, género, lenguaje cultural, motivaciones o conocimientos previos y habilidades que afectarán a sus respuestas y a diferentes formas de presentaciones y representaciones, entre otras. Por tanto, esta dimensión del conocimiento profesional tiene especial relevancia, pues a través de él, el docente trata de un elemento importante para conocer a los alumnos, pues éstos poseen diversos intereses y motivaciones, así como casuísticas diversas, puntos de vista, intereses, pensamientos, entre otros.

Del mismo modo, la valoración de la presencia del conocimiento didáctico del contenido ha dado lugar a unos resultados positivos, pues los alumnos afirman que los profesionales lo desempeñan, en la realidad, la mayoría de las veces. De este modo, la opinión subjetiva de los entrevistados asume la importancia del desempeño de la actividad profesional docente atendiendo y desarrollando este conocimiento. Debemos tener en cuenta que esta dimensión del profesionalismo docente hace que los profesores incorporen aquellos aspectos que se encuentran más relacionados con la enseñanza, por lo que incluye aquellos aspectos propios que permiten transformar el conocimiento científico en enseñable. De esta forma, ha de ser un conocimiento consustancial a todo proceso y tarea educativa.

Tal y como hemos visto, el nivel de importancia otorgado por los alumnos es siempre mayor que la presencia asociada a cada ítem. Resulta un aspecto muy importante a tener en cuenta, pues los alumnos creen, de este modo, que la práctica docente dejaría mucho que desear, pues la valoración o idealización de las dimensiones es siempre superior a la realidad que han observado a lo largo del transcurso de su carrera. A través de la opinión de unos de los principales implicados en la enseñanza, es decir, desde los alumnos, hemos obtenido una visión muy particular que nos avisa y nos lleva a pensar sobre la necesaria renovación y conceptualización de la función del profesorado y el desempeño de su tarea en el aula.
Todas estas puntuaciones han sido obtenidas de estudiantes que cursan el cuarto curso de los distintos grados en educación que oferta la Universidad de Granada. Por tanto, tratamos con personas que ya poseen una madurez conceptual, crítica, reflexiva, terminológica... sobre la educación y, gracias a ello y a su opinión subjetiva, nos aportan una visión muy valiosa desde el punto de vista de los implicados, pues son unas de las personas que pueden valorar mejor la actuación profesional del docente, ya que reciben sus influencias en primera persona y, por supuesto, porque lo hacen desde una perspectiva teórica del conocimiento profesional al cursar una titulación relacionada con la educación.

En la actualidad, hablar de calidad de la educación supone hablar de una serie de aspectos y dimensiones a mejorar de nuestro sistema educativo; entre ellos, se encuentra la figura del docente, pues sobre él recae el peso de formar a las futuras generaciones y, en referencia a nuestro estudio, el conocimiento profesional que contribuya a unas buenas prácticas docentes. Por ello, es necesario hacer un inciso y pararnos a pensar, como profesionales, de lo que estamos mostrando y dando a los demás pues, muchas veces, se produce una seria incongruencia entre lo que mostramos verbalmente, es decir, la teoría, y la práctica que ven nuestros alumnos.

En nuestras manos tenemos la posibilidad del cambio futuro, pues formamos a las nuevas generaciones facilitadoras del saber y éstas, a su vez, a las próximas futuras. De este modo, la influencia de nuestras prácticas con nuestros alumnos van a constituir un gran referente a valorar cuando los futuros educandos desempeñen su tarea profesional. Sin ir más lejos, no existe profesión que no esté más expuesta a las nuevas generaciones trabajadoras que la enseñanza, pues desde que un alumno entra al sistema educativo hasta que decide convertirse él mismo en educador, pasan una serie de años en las que el sujeto está recibiendo grandes influencias y a través de las cuales desarrollará, o tomará como referencia, 
su actuación profesional, especialmente en el transcurso de sus primeros años en la entrada al mundo laboral.

Queremos, por tanto, sensibilizar a todo el colectivo profesional acerca de la necesidad e importancia de la adquisición y desarrollo del conocimiento profesional en la práctica docente, perfeccionándose a lo largo de los años y manteniendo un contacto continuo con la formación durante toda la vida, es decir, la necesaria actualización del docente. Se ha de partir de una postura autocrítica y reflexiva, donde los propios docentes valoren la importancia de su acción, así como la repercusión que tiene ésta en las generaciones futuras y en la mejora de la educación. En definitiva, el conocimiento profesional posee un papel principal, ya que capacita al docente para organizar el contenido académico en enseñanza comprensible para los alumnos, se centra en las características del contexto y de los discentes; pretende adecuar los objetivos y contenidos, entre otras, lo que supone un trabajo reflexivo, estructurado, comprensivo, interactivo, analítico, etcétera.
Para finalizar, llegamos a la conclusión de que la calidad de nuestro sistema educativo depende, en gran medida, de los docentes que lo componen (García-Cano, 2017; Guzmán-Munita, 2017). Hemos comprobado, de acuerdo con la opinión de los entrevistados, que no existe una correlación positiva entre presencia e importancia, pues la segunda se ha dado en mayor cantidad que la primera, según opinan los alumnos de cuarto de grado de la Facultad de Ciencias de la Educación de Granada. La realidad es que, inevitablemente, los docentes influyen en el aprendizaje de los estudiantes y en la mejora de la calidad de la educación. A pesar de la menor presencia del conocimiento profesional docente, el alumnado considera que éste es muy importante para su formación inicial, por lo que albergamos la esperanza de que, aunque los docentes no hayan servido de modelo, en un futuro próximo en el que los alumnos sean profesionales de la educación, sigan siendo firmes con esta concepción y adquieran este conocimiento profesional en su labor docente y, además, que sirva este estudio para concienciar a todo el colectivo educativo. 


\section{Referencias}

Barrón, C. (2015), “Concepciones epistemológicas y práctica docente. Una revisión", REDU, Revista de docencia universitaria, vol. 13, pp. 35-56.

Bazán, S. y S. Zuppa (2016), "El conocimiento didáctico del contenido en la formación inicial del profesorado en historia. Una investigación centrada en las primeras prácticas de aula", Revista de Educación, núm. 9, pp. 225239.

Bermúdez G. y A. L. de Longhi (2012), "El conocimiento didáctico de contenidos biológicos de Ecología”, en R. C. Flores, Experiencias latinoamericanas en educación ambiental, México, GECyTECAEIP, pp. 19-35.

Borrero, M. y S. Losada (2012), "El perfil del buen docente universitario. Una aproximación en función del sexo del alumnado", Revista de Docencia Universitaria, vol.10, pp. 237-249.

Depaepe, F., L. Verschaffel y G. Kelchtermans (2013), "Pedagogical content knowledge: a systematic review of the way in which the concept has pervaded mathematics educational research", Teaching and Teacher Education, vol. 34, núm. 1, pp. 12-35.

Díaz, L. F., Z. Hincapié, M. López y D. Acosta (2016), "Conocimiento didáctico del contenido en maestros en formación del programa de licenciatura en Educación Básica con énfasis en Ciencias Naturales y Educación Ambiental de la Corporación Universitaria del Caribe Sincelejo", Revista Escenarios, vol. 2, núm. 19, pp. 83101.

Fandiño-Parra, Y.-J. (2017), "Formación y desarrollo docente en lenguas extranjeras: revisión documental de modelos, perspectivas y políticas", RIES, Revista Iberoamericana de Educación Superior, vol. 8, núm. 22, pp. 122-143.

Fernández Cruz, M. (2006), Desarrollo profesional docente, Granada, Grupo Editorial Universitario.

García-Cano, M., E. Hinojosa, E. Gutiérrez-Santiuste y M. J. Martínez (2017), "Innovación docente en los grados de educación infantil y primaria. Dando significado y contexto a los aprendizajes de aula en el espacio urbano", Revista de innovación y buenas prácticas docentes, núm. 3, pp. 53-60.

Grossman, P. L. (1990), The making of a teacher: teacher knowledge and teacher education, Nueva York, Teachers College Press.

Guerra, P. y H. Montanegro (2017), "Conocimiento pedagógico: explorando nuevas aproximaciones", Educação e Pesquisa, vol. 43, núm. 3, pp. 663-680.

Guzmán-Munita, M. (2017), "Concepción didáctica de competencias para profesores de castellano", en RIES, Revista Iberoamérica de Educación Superior, vol. 8, núm. 22, pp. 25-44.

Köing, J., S. Blömeke, P. Klein, A. Busse y K. Gabriele (2014), "Is teacher's general pedagogical knowledge a premise for noticing and interpreting classroom situations? A video bases assessment approach", Teaching and Teacher Education, vol. 38, pp. 76-88.

Latorre Medina, M. J., M. G. López López Y M. J. Rodríguez Entrena(2009), "El profesorado universitario ante el Espacio Europeo de Educación Superior", en E. Leclercq y D. Niclot, Former des professionnels de la formation en europe, Reims, EPURE (Éditions et Presses Universitaires de Reims), pp. 77-108.

López-López, M. C. (2009), "El papel de la universidad en la formación para el ejercicio profesional", en J. Tejada Fernández (coord.), Estrategias de innovación en la formación para el trabajo, Madrid, Tornapunta Ediciones, pp. 417-427.

Marcelo, C. (2014), "Estudio sobre estrategias de inserción profesional en Europa", Revista Iberoamericana de Educación, <http://rieoei.org/oeivirt/rie19a03.htm> [Consulta: octubre de 2017].

Marcelo, C. y D. Vaillant (2015), Desarrollo profesional docente. ¿Cómo se aprende a enseñar?, Madrid, NARCEA.

Osorio, D., Y. Hernández, R. Soto y Y. Orozco (2014), "El conocimiento del contexto como un conocimiento profesional del profesor de biología. Aportes de cuatro docentes y sus experiencias", <http://revistas. pedagogica.edu.co/index.php/bio-grafia/article/ 
view/2484> [Consulta: noviembre de 2017].

Rodríguez, D. y J. Valldeoriola (2009), Metodología de la Investigación, Barcelona, FUOC.

Shulman, L. S. (1999), "Foreword", en J. Gess-Newsome y N. G. Lederman (eds.), Examining pedagogical content knowledge: the construct and its implications for science teaching, Dordrecht, The Netherlands, Kluwer Academic Publishers, pp. ix-xii.

Shulman, L. S. y J. H. Shulman (2004), "How and what teachers learn: a shifting perspective", fournal of Curriculum Studies, vol. 36, núm. 2, pp. 257-271.

Shulman, L. S. (2005), "Conocimiento y enseñanza: fundamentos de la nueva reforma", Profesorado, Revista de curriculum y formación del profesorado, vol. 9, núm. 2, pp. 1-30.

Solís, E., R. Porlán y A. Rivero (2012), “CCómo representar el conocimiento curricular de los profesores de ciencias y su evolución?”, Enseñanza de las Ciencias. Revista de Investigación y Experiencias Didácticas, vol. 30, núm. 3, pp. 9-30.

Stabback, P. (2016), "Qué hace a un currículo de calidad", Oficina Internacional de Educación de la UNESCO, <http://unesdoc.unesco.org/ images/0024/002439/243975s.pdf> [Consulta: noviembre de 2017].

Stenhouse, L. (2010), Investigación y desarrollo del curriculum, Madrid, Morata.

Vera, J. Á., L. E. Torres y E. E. Martínez (2014), "Evaluación de competencias básicas en TIC en docentes de educación superior en México", Revista de Medios y Educación, núm. 44, pp. 143-155.

Vergara, G. y H. Cofré (2014), "Conocimiento pedagógico del contenido: ¿el paradigma perdido en la formación inicial y continua de profesores en Chile?", Estudios Pedagógicos, Valdivia, vol. 40, núm. especial, pp. 323-328. Vicedo, A. (2015), “‘Quién debe enseñar qué cosa en educación médica?' El papel del profesor y el conocimiento pedagógico del contenido", Revista Educación Médica Superior, <http://scielo.sld.cu/scielo. php?pid=S0864 $-\quad 21412015000300001 \&$ script $=$ sci_ arttext\&tlng $=$ pt $>$ [Consulta: octubre de 2017].

\section{Cómo citar este artículo:}

Rodríguez-García, Antonio-Manuel y Nazaret Martínez-Heredia (2019), "Conocimiento profesional del docente en educación superior: una confrontación entre lo real y lo deseado", , en Revista Iberoamericana de Educación SUperior (RIES), México, UNAM-IISUE/ Universia, vol. x, Núm. 29, pp. 25-41, DoI: https://doi.org/10.22201/iisue.20072872e.2019.29.521 [consulta: fecha de última consulta]. 